\title{
Antibacterial Activity of Copper Nanoparticles Synthesized by Bambusa arundinacea Leaves Extract
}

\author{
Jayarambabu Naradala ${ }^{1}$, , Akshaykranth Allam 1(i), Venkatappa Rao Tumu 1* $^{(i)}$, Rakesh Kumar \\ Rajaboina ${ }^{1}$ (D) \\ 1 Department of Physics, National Institute of Technology Warangal, Telangana State-506004, India \\ * Correspondence: tvraokmm@nitw.ac.in;
}

Scopus Author ID24281986900

Received: 23.02.2021; Revised: 8.04.2021; Accepted: 12.04.2021; Published: 26.04.2021

\begin{abstract}
A novel bio-fabrication of copper nanoparticles (CuNPs) using Bambusa arundinacea leaves aqueous extract solution, and their prospective biological activities are reported. Scanning and Transmission Electron Microscopy study of synthesized powder confirms the formation of Cu NPs with a size range 15-30 $\mathrm{nm}$. The Dynamic Light Scattering (DLS) study further confirms the average particle size as $24.4 \mathrm{~nm}$, and the observed zeta potential value of $-16.1 \mathrm{mV}$ affirms the high stability of Cu NPs. The synthesized $\mathrm{Cu}$ NPs exhibited potent antibacterial activity against Escherichia coli, Staphylococcus aureus, Bacillus subtilis, and Proteus vulgaris. This method provides a safe, clean, eco-friendly, and economical way to produce $\mathrm{Cu}$ NPs.
\end{abstract}

Keywords: green synthesis; nanoparticles; electron microscopy; antibacterial activity.

(C) 2021 by the authors. This article is an open-access article distributed under the terms and conditions of the Creative Commons Attribution (CC BY) license (https://creativecommons.org/licenses/by/4.0/).

\section{Introduction}

In recent years, the green synthesis of nanoparticles has been attracted much attention due to its reliable, sustainable, eco-friendly, cost-effective, and non-toxic protocol [1,2]. The main drawbacks of thermal, physical, and chemical methods of nanoparticle preparation are the use of sophisticated instruments, high-temperature synthesis, toxic solvents, and quite expensive. The main advantages of the green chemistry method over chemical and physical approaches are simple to control the reaction, cost-effective, low-temperature synthesis, nontoxic chemicals, easy to scale-up, good production rate, and eco-friendly. The green synthesis was used to prepare different metal oxide and metal nanoparticles in the literature [3]. Among all metal nanoparticles, copper $(\mathrm{Cu})$ nanoparticles are superior due to their unique chemical and physical properties, such as high thermal conductivity, electrical conductivity, and biological activity. Copper is highly toxic to organisms and non-toxic to animal cells, and it is considered a useful bactericidal metal. Copper NPs have been used in different applications such as health, food, medical, consumer, and some industrial sectors. In this manuscript, green synthesis of $\mathrm{Cu}$ NPs with a novel plant extract is reported for the first time to the best of our knowledge.

The green synthesis of $\mathrm{Cu}$ NPs has been a promising research area in biomedicine, biosensors, and pharmacy in recent times. Green synthesis of $\mathrm{Cu}$ NPs focuses on the employment of fungi, bacteria, and plant extracts, as reducing and capping agents [4-6]. Among all, nanoparticle preparation with plant extracts has been attracted massive attention due to the accessibility of a biological entity. The different plant extracts such as Azadirachta indica, 
Cissusarnotiana, Green and black tea, Eichhornia crassipes, Falcaria vulgaris, Millettiapinnata, Perseaa mericana, Quisqualis indica, Ziziphusspina-christi were reported for the $\mathrm{Cu}$ NPs synthesis in recent years [7]. To the best of the authors' knowledge, Cu NPs synthesis with Bambusa plant leaves (BPL) extract is not reported. The BPL extract holds natural bio-compounds like flavonoids, tannins, aldehydes, ketones, alkaloids, saponins, polysaccharides, and additional nutritional compounds [8,9]. In this green synthesis, the plant extract act as a strong natural reducing agent and stabilizer for $\mathrm{Cu}$ NPs. The phytoconstituents like flavonoids, phenolic acids, tannins, saponins, glycoside, alkaloid, and polysaccharides are responsible for reducing $\mathrm{Cu}$ metal salts into $\mathrm{Cu}$ NPs. This process's main advantages are (1) easy mixing of extract solution with an aqueous solution of metal salt precursor; (2) prepared $\mathrm{Cu}$ NPs are less toxic and biocompatible. The surface of the prepared $\mathrm{Cu}$ NPs has some bonded biomolecules; thus, they show good antibacterial activity against pathogenic bacteria.

In the present study, we have used BPL extract to synthesize $\mathrm{Cu}$ NPs and evaluated their antibacterial activity against four pathogenic bacteria: Escherichia coli, Staphylococcus aureus, Bacillus subtilis, and Proteus vulgaris.

\section{Materials and Methods}

\subsection{Materials.}

The Bambusa leaves were collected from in and around the campus of the National Institute of Technology Warangal, India. The Cupric acetate $\left(\mathrm{Cu}\left(\mathrm{CH}_{3} \mathrm{COO}\right)_{2}\right)$ procured from Sigma Aldrich was used without further purification. The bacterial cultures of Escherichia coli (MTCC-433), Staphylococcus aureus (MTCC-1430), Bacillus subtilis (MTCC-441), and Proteus vulgaris (MTCC-426) were obtained from the Microbial Type Culture Collection and Gene Bank (MTCC) Chandigarh, India.

\subsection{Bambusa arundinacea leaves extract preparation.}

The Bambusa leaves were washed several times with double distilled water and dried at room temperature. The leaves were cut into small pieces and put into the glass beaker containing $100 \mathrm{ml}$ distilled water. The leaves extract was generated by boiling the leaves in the water for 30 minutes at $80^{\circ} \mathrm{C}$. The extract was filtered with the help of Whatman filter paper. The collected, filtered extract solution was stored for further usage for $\mathrm{Cu}$ NPs synthesis.

\subsection{Biosynthesis of $\mathrm{Cu} N P$ s.}

Initially, Bambusa arundinacea leaves extract was prepared. Subsequently, cupric acetate $0.01 \mathrm{M}$ solution was prepared in a beaker at $60^{\circ} \mathrm{C}$. In the next step, $25 \mathrm{ml}$ of aqueous extract of Bambusa leaves was added to the cupric acetate solution. The resulting solution was stirred at $65^{\circ} \mathrm{C}$ for four hours to get the $\mathrm{Cu}$ NPs. The color of the solution has been started to change from blue to bluish-green after $30 \mathrm{~min}$ of reaction, indicating the formation of Cu NPs [10-12]. The obtained powder was washed with an ethanol solution to remove unwanted compounds. The resulting powder was characterized by different techniques.

\subsection{Characterization techniques of $\mathrm{Cu} N \mathrm{NP}$.}

The morphology and size of the prepared $\mathrm{Cu}$ NPs were analyzed with the scanning electron microscope (SEM, Zeiss) and transmission electron microscope (TEM, JEOL-2100). 
The crystalline nature of the $\mathrm{Cu}$ NPs powder was confirmed by X-ray diffraction (XRD, Bruker D8 advance). UV-spectrophotometer was used for confirmation of the $\mathrm{Cu}$ NPs formation. Particle size distribution, the zeta potential, and polydispersity index (PDI) of Cu NPs were measured by particle size analyzer (Horibha SZ-100). FTIR spectrometer was used to determine the functional chemical groups (Perkin Elmer-400).

\subsection{Antibacterial activity.}

The antibacterial activity of green synthesized $\mathrm{Cu}$ NPs was analyzed using the Disc diffusion method. The bacterial inoculums were firstly grown in LB broth for $24 \mathrm{~h}$ at $28^{\circ} \mathrm{C}$ and $200 \mathrm{rpm}$. It was then re-suspended in LB medium until the optical density (OD) is adjusted to 0.1 at $600 \mathrm{~nm}$, which corresponds to $10^{8}$ Colony Forming Units (CFU)/mL. Spread plate technique was used in which $100 \mu \mathrm{L}$ of each bacterial suspension was spread over nutrient agar medium. A set of four concentrations $(50,100,150$, and $200 \mu \mathrm{L})$ of $\mathrm{Cu}$ NPs colloidal solution in the disc were prepared and used in the study. The four different concentrations of the $\mathrm{Cu}$ NPs in respective discs were placed over the lawn of bacterial culture and standard. All the agar plates were incubated in the bacteriological incubator for $24 \mathrm{~h}$ at $37^{\circ} \mathrm{C}$. The zone of inhibition (ZOI) around the discs was determined with a measuring scale (mm).

\section{Results and Discussion}

\subsection{Characterization of $\mathrm{Cu} N \mathrm{NP}$.}

The FESEM image of the obtained powder shown in figure 1(a) confirms the formation of $\mathrm{Cu}$ NPs and most of the particles agglomerated in nature. Further, TEM was employed to get information about the shape and size of the NPs. It is evident from figure 1(b), the synthesized $\mathrm{Cu}$ NPs are spherical with smooth surfaces. The histogram shown in the inset of figure 1(b) represents the particle size distribution and indicating that the average size of $\mathrm{Cu}$ NPs is $23 \mathrm{~nm}$. The size of the $\mathrm{Cu}$ NPs dependent on the plant extract concentrations, the phytochemicals involved in the formation of $\mathrm{Cu}$ NPs.
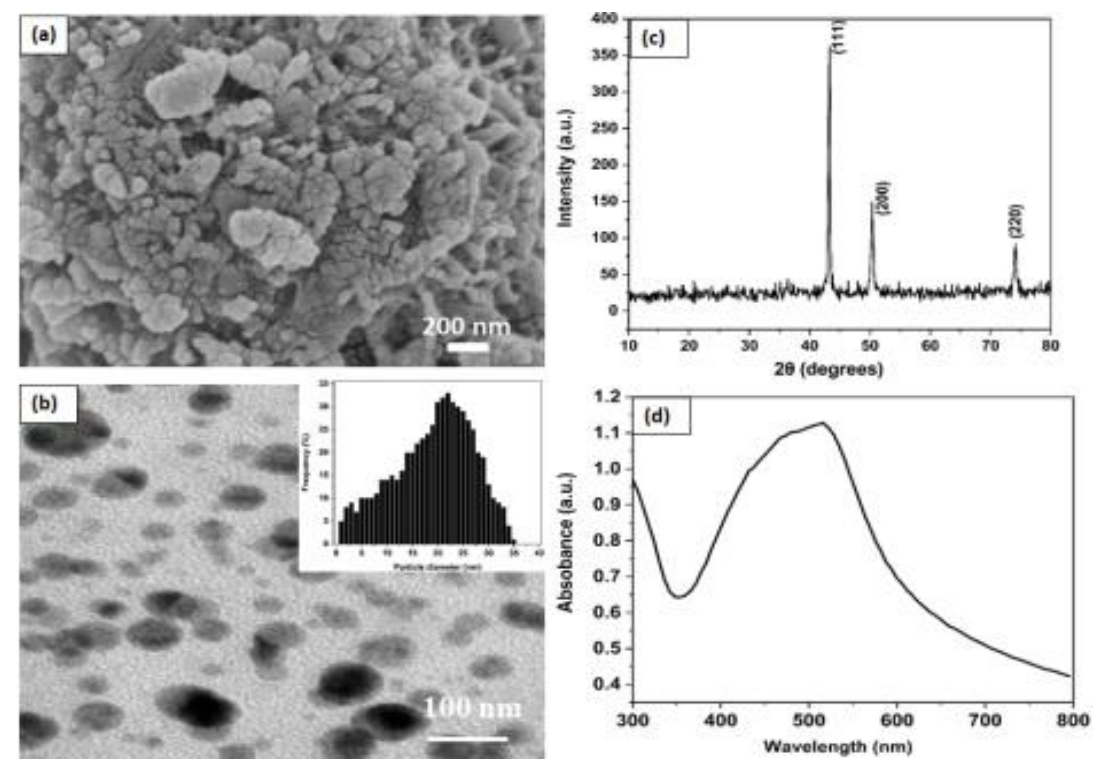

Figure 1. (a) FESEM image of green synthesized Cu NPs, (b) TEM image of the Cu NPs, Inset of (b) histogram of particle size distribution, (c) XRD pattern of the Cu NPs powder, (d)UV-Vis absorption spectra of the $\mathrm{Cu}$ NPs. 
The crystalline characteristic of synthesized $\mathrm{Cu}$ NPs powder was analyzed by XRD. The XRD pattern was recorded in the range from $10^{\circ}$ to $80^{\circ}$ and shown in Figure 1 (c). In the XRD pattern, three diffraction maxima were observed at $2 \theta=43.35^{\circ}, 50.50^{\circ}$, and $74.21^{\circ}$. The diffraction maxima's were indexed as (111), (200), and (220) plane of face center cubic structure of $\mathrm{Cu}$, respectively. The peaks observed in the XRD pattern were matched with JCPDS card number 04-0836[13]. Figure 2(d) shows the UV-Visible absorption spectra of the colloidal suspension of $\mathrm{Cu}$ NPs in methanol solution. The surface plasmon resonance (SPR) absorption band is observed at $510 \mathrm{~nm}$ due to the combined vibration of electrons of metal nanoparticles in resonance with a light wave. The observed broad absorption band is due to the wide size distribution of copper nanoparticles. The size, shape of the nanoparticles, solvents, and reducing agents employed for the synthesis strongly affect the surface-plasmon band.

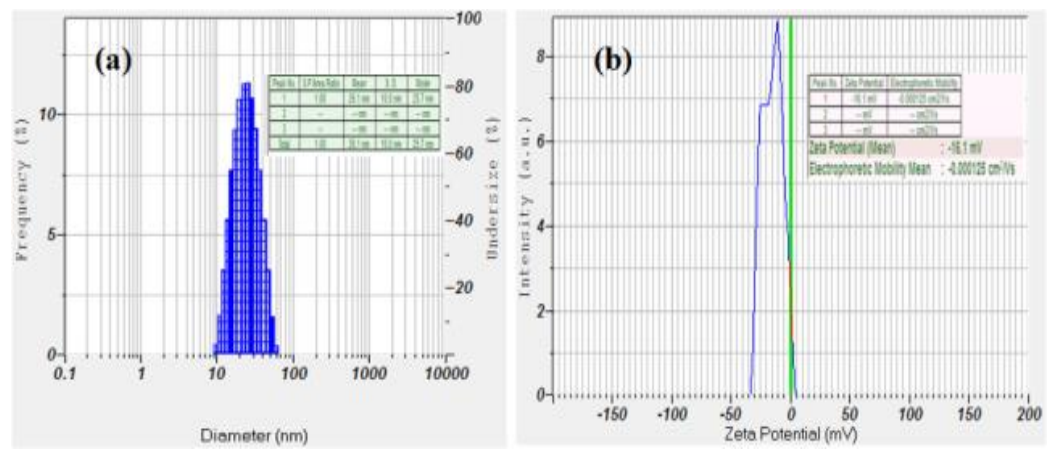

Figure 2. (a) Particles size distribution, (b) zeta potential analysis of bio-synthesized Cu NPs.

Further, the size and distribution of $\mathrm{Cu}$ NPs were determined by dynamic light scattering. Figure 2(a) shows the size distribution of $\mathrm{Cu}$ NPs dispersed in ethanol. At the peak position, the Z-average diameter of bio-synthesized $\mathrm{Cu}$ NPs was $\sim 24.4 \mathrm{~nm}$ with a polydispersity index (PDI) of 0.0018. The zeta potential analysis commonly assesses the stability of nanoparticles. The zeta potential values of NPs with values $>+25 \mathrm{mV}$ or $<-25 \mathrm{mV}$ usually have a high degree of stability. The zeta potential value of the $\mathrm{Cu}$ NPs was found to be $-16.1 \mathrm{mV}$, as shown in figure (b), and confirms the stability of the nanoparticles. The negative Zeta potential value shown by biosynthesized $\mathrm{Cu}$ NPs may be due to the presence of bioorganic components in the extract as capping agents $[13,14]$.

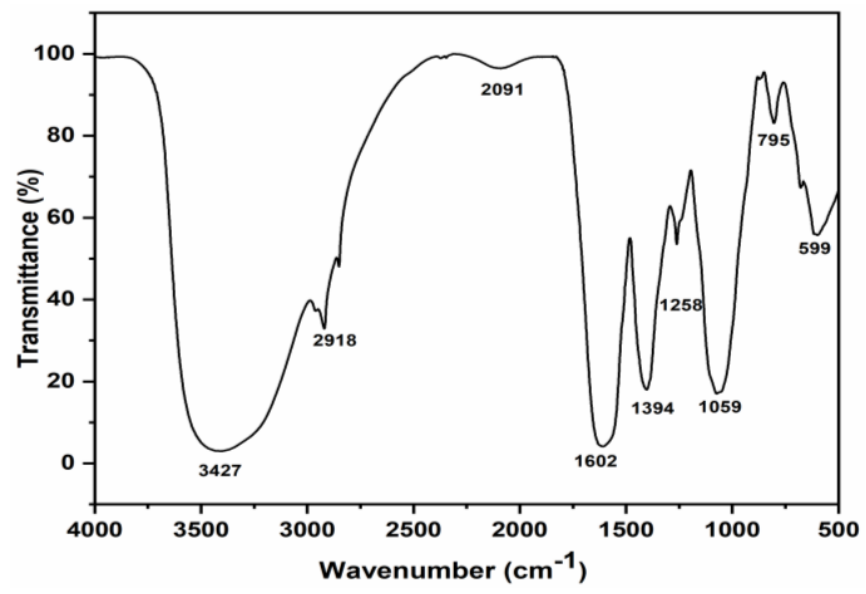

Figure 3. FTIR analysis of biosynthesized $\mathrm{Cu}$ NPs.

FTIR spectra analysis was used to determine the biologically active compound molecules present in the $\mathrm{Cu}$ NPs powder. In the FTIR spectra in Figure 3, absorption peaks 
are observed at 3427, 2918, 2091, 1602, 1394, 1258, 1059, 795 and $599 \mathrm{~cm}^{-1}$. The peaks at 2918,1602 , and $1059 \mathrm{~cm}^{-1}$ indicate the $\mathrm{C}-\mathrm{H}$ asymmetric stretching, $\mathrm{C}=\mathrm{O}$ aromatic vibrations, and $\mathrm{C}=\mathrm{C}$ stretching, respectively [15]. The peak at $2091 \mathrm{~cm}^{-1}$ confirms the carboxylate ions [16]. The peak positions at 1394 and $1258 \mathrm{~cm}^{-1}$ correspond to the organic and aromatic molecule derivatives like phenolic, alkaloids, flavonoids, tannins, aldehydes, and ketones present in the plant extract the peaks at 795 and $599 \mathrm{~cm}^{-1}$ identified as the aromatic ring of amino acids. The peak observed at $3427 \mathrm{~cm}^{-1}$ may be assigned to $\mathrm{OH}$ or $\mathrm{NH}$ stretching of phenolic compound. The involvement of phytochemicals in the formation of $\mathrm{Cu}$ NPs is confirmed with the FTIR analysis. Hence we can conclude these phytochemicals play a significant role in synthesizing Cu NPs [17].

\subsection{Antibacterial activity of $\mathrm{Cu} N \mathrm{~N}$.}

Figures 4(a)-(d) show the antibacterial activity of different concentrations of Cu NPs against four pathogenic bacteria of Proteus vulgaris, Escherichia coli, Staphylococcus aureus, and Bacillus subtilis, respectively. The central white spot in figures 4 (a)-(d) is the standard sample used as reference measurement. The measured zones of inhibition diameter (in $\mathrm{mm}$ ) around the discs were summarized for different concentrations of $\mathrm{Cu}$ NPs shown in figure 4(e). The diameter of the zone of inhibition gives information about the magnitude of the susceptibility of microorganisms. It is observed that $\mathrm{Cu}$ NPs demonstrated a high degree of antibacterial activity for Escherichia coli and Bacillus subtilis. Whereas, in the case of Proteus vulgaris and Staphylococcus aureus, moderate activity is observed for Cu NPs.
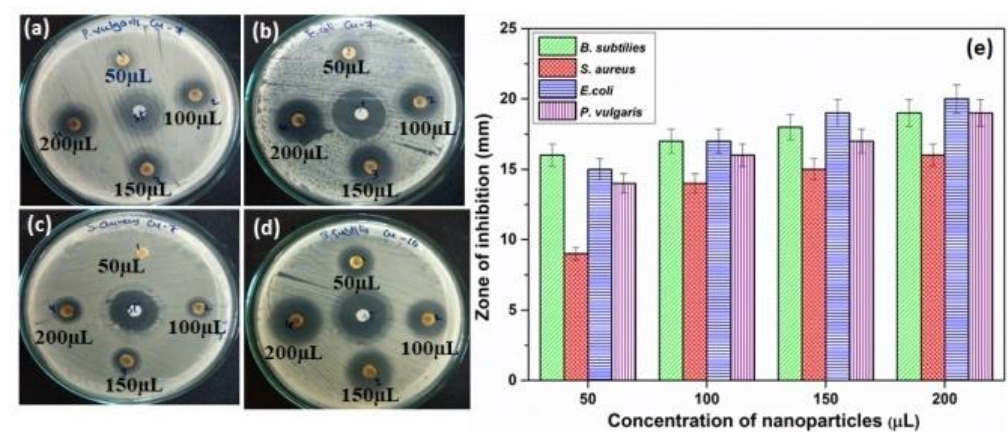

Figure 4. Antibacterial activity of $\mathrm{Cu}$ nanoparticles at different concentrations against (a) Proteus vulgaris, (b) Escherichia coli, (c) Staphylococcus aureus, (d) Bacillus subtilis, (e) graphical representation of zone of inhibition values at different concentration of Cu NPs.

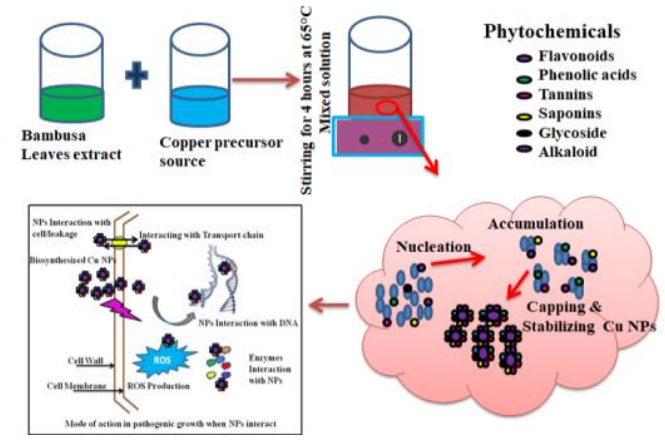

Figure 5. Schematic diagram of $\mathrm{Cu}$ nanoparticles synthesis and antibacterial activity.

There are different accepted mechanisms for the antibacterial activity of metal nanoparticles $[18,19]$. The most-reported mechanism is based on the reactive oxygen species generation (ROS). $\mathrm{Cu}$ NPs also dramatically enhance the cellular ROS level that influences 
lipid peroxidation, protein oxidation, and DNA destruction and finally kills the microorganism cells [20-22]. The ROS contain radical compounds such as hydroxyl (-OH), superoxide radical $\left(\mathrm{O}_{2}{ }^{-}\right)$, singlet oxygen $\left({ }^{1} \mathrm{O}_{2}\right)$, and hydrogen peroxide $\left(\mathrm{H}_{2} \mathrm{O}_{2}\right)$, which destroy the bacteria. To conclude, the green synthesized $\mathrm{Cu}$ NPs demonstrated significant antibacterial activity against various organisms. The schematic diagram of the biosynthesis of $\mathrm{Cu}$ NPs using Bambusa arundinacea aqueous extract and antibacterial activity is shown in figure 5.

\section{Conclusions}

In summary, Bambusa arundinacea aqueous extract was used successfully to synthesize the $\mathrm{Cu}$ NPs for the first time. The synthesized nanoparticles were characterized by using SEM, TEM, XRD, UV-Visible, and DLS techniques. The synthesized nanoparticles were spherical in shape and crystalline in nature, with an average particle size $24.4 \mathrm{~nm}$. The green synthesized $\mathrm{Cu}$ NPs exhibited excellent antibacterial activity against Escherichia coli, Staphylococcus aureus, Bacillus subtilis, and Proteus vulgaris. The antibacterial activity was also tested for different concentrations of the $\mathrm{Cu}$ NPs and found increased activity with the increase of concentration. Further, the application of green synthesized Cu NPs can be extended to antioxidant, anticancer, and wound healing activities.

\section{Funding}

Maharashtra Bamboo Development Board (MBDB), Nagpur (Grant No: MD/MBDB/CR86/18-19/589).

\section{Acknowledgments}

The authors acknowledge the financial support from Maharashtra Bamboo Development Board (MBDB), Nagpur (Grant No: MD/MBDB/CR-86/18-19/589). The authors also sincerely acknowledge the encouragement given by Sri T. Sai Kumar Reddy, MD, MBDB, Nagpur, to carry out this work.

\section{Conflicts of Interest}

The authors declare no conflict of interest.

\section{References}

1. Singh, A.; Gautam, P.K.; Verma, A.; Singh, V.; Shivapriya, P.M.; Shivalkar, S.; Sahoo, A.K.; Samanta, S.K. Green synthesis of metallic nanoparticles as effective alternatives to treat antibiotics resistant bacterial infections: A review. Biotechnology Reports 2020, 25, https://doi.org/10.1016/j.btre.2020.e00427.

2. Shafey, A.M.E. Green synthesis of metal and metal oxide nanoparticles from plant leaf extracts and their applications: A review. Green Processing and Synthesis 2020, 9, 304-339, https://doi.org/10.1515/gps-20200031.

3. Thakore, S.I.; Nagar, P.S.; Jadeja, R.N.; Thounaojam, M.; Devkar, R.V.; Rathore, P.S. Sapota fruit latex mediated synthesis of $\mathrm{Ag}, \mathrm{Cu}$ mono and bimetallic nanoparticles and their in vitro toxicity studies. Arabian Journal of Chemistry 2019, 12, 694-700, https://doi.org/10.1016/j.arabjc.2014.12.042.

4. Sharma, P.; Pant, S.; Dave, V.; Tak, K.; Sadhu, V.; Reddy, K.R. Green synthesis and characterization of copper nanoparticles by Tinospora cardifolia to produce nature-friendly copper nano-coated fabric and their antimicrobial evaluation. Journal of Microbiological Methods 2019, 160, 107-116, https://doi.org/10.1016/j.mimet.2019.03.007.

5. Zangeneh, M.M.; Ghaneialvar, H.; Akbaribazm, M.; Ghanimatdan, M.; Abbasi, N.; Goorani, S.; Pirabbasi, E.; Zangeneh, A. Novel synthesis of Falcaria vulgaris leaf extract conjugated copper nanoparticles with potent cytotoxicity, antioxidant, antifungal, antibacterial, and cutaneous wound healing activities under in 
vitro and in vivo condition. Journal of Photochemistry and Photobiology B: Biology 2019, 197, https://doi.org/10.1016/j.jphotobiol.2019.111556.

6. Ismail, M.; Gul, S.; Khan, M.I.; Khan, M.A.; Asiri, A.M.; Khan, S.B. Green synthesis of zerovalent copper nanoparticles for efficient reduction of toxic azo dyes congo red and methyl orange. Green Processing and Synthesis 2019, 8, 135-143, https://doi.org/10.1515/gps-2018-0038.

7. Thiruvengadam, M.; Chung, I.-M.; Gomathi, T.; Ansari, M.A.; Gopiesh Khanna, V.; Babu, V.; Rajakumar, G. Synthesis, characterization and pharmacological potential of green synthesized copper nanoparticles. Bioprocess and Biosystems Engineering 2019, 42, 1769-1777, https://doi.org/10.1007/s00449-019-02173-y.

8. Rajeshkumar, S.; Menon, S.; Venkat Kumar, S.; Tambuwala, M.M.; Bakshi, H.A.; Mehta, M.; Satija, S.; Gupta, G.; Chellappan, D.K.; Thangavelu, L.; Dua, K. Antibacterial and antioxidant potential of biosynthesized copper nanoparticles mediated through Cissus arnotiana plant extract. Journal of Photochemistry and Photobiology B: Biology 2019, 197, https://doi.org/10.1016/j.jphotobiol.2019.111531.

9. Asghar, M.A.; Asghar, M.A. Green synthesized and characterized copper nanoparticles using various new plants extracts aggravate microbial cell membrane damage after interaction with lipopolysaccharide. International Journal of Biological Macromolecules 2020, 160, 1168-1176, https://doi.org/10.1016/j.ijbiomac.2020.05.198.

10. Yaqub, A.; Malkani, N.; Shabbir, A.; Ditta, S.A.; Tanvir, F.; Ali, S.; Naz, M.; Kazmi, S.A.R.; Ullah, R. Novel Biosynthesis of Copper Nanoparticles Using Zingiber and Allium sp. with Synergic Effect of Doxycycline for Anticancer and Bactericidal Activity. Current Microbiology 2020, 77, 2287-2299, https://doi.org/10.1007/s00284-020-02058-4.

11. Rajesh, K.M.; Ajitha, B.; Ashok Kumar Reddy, Y.; Suneetha ,Y.; SreedharaReddy. P, Assisted green synthesis of copper nanoparticles using Syzygium aromaticum bud extract: Physical, optical and antimicrobial properties, Optik 2018, 154, 593-600, https://doi.org/10.1016/j.ijleo.2017.10.074.

12. Sadaf N.; Ziaullah S.; Aneela J.; Amjad A.; Syed Bilal H.; Sidra Z.; Hazrat A.; Syed Aun, M, A fungal based synthesis method for copper nanoparticles with the determination of anticancer, antidiabetic and antibacterial activities. Journal of Microbiological Methods 2020, 174, 105966-10579.

13. Jahan, I.; Erci, F.; Isildak, I. Facile microwave-mediated green synthesis of non-toxic copper nanoparticles using Citrus sinensis aqueous fruit extract and their antibacterial potentials. Journal of Drug Delivery Science and Technology 2021, 61, https://doi.org/10.1016/j.jddst.2020.102172.

14. Jahan, I.; Erci, F.; Isildak, I. Microwave-Assisted Green Synthesis of Non-Cytotoxic Silver Nanoparticles Using the Aqueous Extract of Rosa santana (rose) Petals and Their Antimicrobial Activity. Analytical Letters 2019, 52, 1860-1873, https://doi.org/10.1080/00032719.2019.1572179.

15. Medina Cruz, D.; Tien-Street, W.; Zhang, B.; Huang, X.; Vernet Crua, A.; Nieto-Argüello, A.; CholulaDíaz, J.L.; Martínez, L.; Huttel, Y.; González, M.U.; García-Martín, J.M.; Webster, T.J. Citric juicemediated synthesis of tellurium nanoparticles with antimicrobial and anticancer properties. Green Chemistry 2019, 21, 1982-1998, https://doi.org/10.1039/C9GC00131J.

16. Jahan, I.; Isildak, I. Microwave Irradiation System For A Rapid Synthesis Of Non-Toxic Metallic Copper Nanoparticles From Green Tea. Trakya University Journal of Natural Sciences 2020, 21, 79-86, https://doi.org/10.23902/trkjnat.731692.

17. Jahan, I.; Erci, F.; Cakir-Koc, R.; Isildak, I. Microwave-irradiated green synthesis of metallic silver and copper nanoparticles using fresh ginger (Zingiber officinale) rhizome extract and evaluation of their antibacterial potentials and cytotoxicity. Inorganic and Nano-Metal Chemistry 2021, 51, 722-732, https://doi.org/10.1080/24701556.2020.1808017.

18. Erci, F.; Cakir-Koc, R.; Yontem, M.; Torlak, E. Synthesis of biologically active copper oxide nanoparticles as promising novel antibacterial-antibiofilm agents. Preparative Biochemistry \& Biotechnology 2020, 50, 538-548, https://doi.org/10.1080/10826068.2019.1711393.

19. Wu, S.; Rajeshkumar, S.; Madasamy, M.; Mahendran, V. Green synthesis of copper nanoparticles using Cissus vitiginea and its antioxidant and antibacterial activity against urinary tract infection pathogens. Artif Cells Nanomed Biotechnol 2020, 48, 1153-1158, https://doi.org/10.1080/21691401.2020.1817053.

20. Mali, S.C.; Dhaka, A.; Githala, C.K.; Trivedi, R. Green synthesis of copper nanoparticles using Celastrus paniculatus Willd. leaf extract and their photocatalytic and antifungal properties. Biotechnology Reports 2020, 27, https://doi.org/10.1016/j.btre.2020.e00518.

21. Gour, A.; Jain, N.K. Advances in green synthesis of nanoparticles. Artificial Cells, Nanomedicine, and Biotechnology 2019, 47, 844-851, https://doi.org/10.1080/21691401.2019.1577878.

22. Ali, J.S.; Mannan, A.; Nasrullah, M.; Ishtiaq, H.; Naz, S.; Zia, M. Antimicrobial, antioxidative, and cytotoxic properties of Monotheca buxifolia assisted synthesized metal and metal oxide nanoparticles. Inorganic and Nano-Metal Chemistry 2020, 50, 770-782, https://doi.org/10.1080/24701556.2020.1724150. 